\title{
Retraso en el diagnóstico como factor pronóstico de discapacidad en pacientes con lepra en Paraguay. Estudio de casos y controles
}

\author{
Delay in diagnosis as a prognostic factor for disability in patients with leprosy in \\ Paraguay. Case and control study
}

María Margarita Aguilera', Lourdes Raquel Samaniego y yargarita Samudio ${ }^{3}$

'Universidad Nacional de Asunción. UNA Facultad de Ciencias Químicas.

¿Universidad Nacional de Asunción. UNA. Facultad de Ciencias Químicas Departamento de Farmacia.

${ }^{3}$ Instituto de Investigaciones en Ciencias de la Salud. Asunción, Paraguay.

Los autores declaran no tener conflicto de intereses.

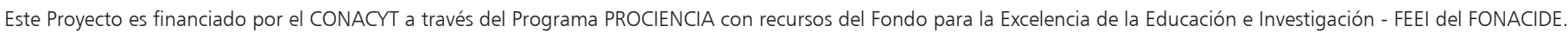

Recibido: 27 de octubre de 2020 (segunda versión: 6 de junio de 2021) / Aceptado: 12 de julio de 2021

\section{Resumen}

Introducción: La lepra, una infección crónica, es una de las mayores causas de discapacidad prevenible. El inicio temprano del tratamiento previene el desarrollo de discapacidad. Objetivo: Identificar los factores pronóstico de discapacidad en individuos con lepra multibacilar y paucibacilar que culminaron el tratamiento farmacológico entre el 2011 y 2017 en Paraguay. Pacientes y Métodos: Se realizó un estudio de casos y controles, con 34 pacientes, 9 casos, 25 controles. Los casos fueron pacientes con discapacidad Grado 1 que presentaban falta de sensibilidad en miembros inferiores o superiores, y los de Grado 2, lagoftalmos, rigidez, ulceraciones, garra pasiva, garra activa. Los controles no presentaron discapacidad. Resultados: La edad media de los pacientes fue $53 \pm 15,2$ años, el $55,9 \%$ fue de sexo masculino y $58,9 \%$ tenía educación primaria o no tenía educación formal. El 58,8\% de los pacientes presentó lepra multibacilar; y el $64,7 \%$ fue diagnosticado tras consultar con dos o más médicos. Retraso en el diagnóstico mayor a un año fue significativamente $(\mathrm{p}=0,047)$ mayor en los casos que en los controles (77,8 vs 12\%; OR: 7,44; IC95\%: 1,02-67,86). Conclusión: El retraso en el diagnóstico mayor a un año es un factor pronóstico de discapacidad.

Palabras clave: lepra; retraso diagnóstico; discapacidad; Paraguay.

\begin{abstract}
Background: Leprosy, a chronic infectious disease, is one of the major causes of preventable disability. Early treatment prevents neurological damage and disability. Aim: To identify prognostic factors of disability in individuals with multibacillary and paucibacillary leprosy who completed a drug treatment between 2011 and 2017 in Paraguay. Methods: A case-control study was carried out on 34 patients, of them 9 were cases and 25 controls. Cases were those patients with Grade 1, presented lack of sensation in lower or upper limbs, and those of Grade 2 lagophthalmos, rigidity, visible deformity ulcerations, passive claw, active claw. Controls had no disabilities. Results: Mean age of the patients was $53 \pm 15.2$ years, $55.9 \%$ were male, and $58.9 \%$ had primary education or no formal education. Multibacillary leprosy was found in $58.8 \%$ of patients; and $64.7 \%$ were diagnosed after consulting with two or more physicians. Diagnosis delay of more than one year was significantly $(\mathrm{p}=0.047)$ greater in the cases than in the controls $(77.8 \%$ vs $12 \%$; OR: 7.44 ; 95\% CI: 1.02-67.86). Conclusion: In this study, a diagnosis delay of more than one year is a prognostic factor for disability.

Keywords: leprosy; diagnostic delay; disability; Paraguay.
\end{abstract}


multibacilar y paucibacilar que culminaron el tratamiento farmacológico entre el 2011 y 2017.

\section{Pacientes y Métodos}

Se planteó un diseño de casos y controles para identificar si el retraso en el diagnóstico es un factor pronóstico para el desarrollo de la discapacidad.

\section{Criterios de inclusión}

Pacientes mayores de edad, de ambos sexos que culminaron el tratamiento farmacológico entre 2011 y 2017, pertenecientes a los distritos de Capiatá, Fernando de la Mora, San Lorenzo, Luque y Lambaré del Departamento Central, que accedieron a participar del estudio y firmaron el consentimiento informado.

\section{Criterios de exclusión}

Pacientes embarazadas, individuos con patologías como tuberculosis, síndrome de inmunodeficiencia adquirida $\mathrm{u}$ otras dolencias con tratamiento farmacológico y enfermos mentales.

Se establecieron los casos y controles en base a la presencia de discapacidad. Los casos fueron aquellos pacientes con Grado 1 (G1) y Grado 2 (G2), y los controles aquellos sin discapacidad, Grado 0 (G0). Los pacientes con Grado 1 (G1) presentaban falta de sensibilidad en miembros inferiores o superiores, y los de Grado 2 (G2) lagolftalmos, deformidad visible, ulceraciones en miembros inferiores, garra pasiva, garra activa.

En Paraguay, el Programa Nacional de Control de Lepra coordina las acciones relativas a la enfermedad en todas las Regiones Sanitarias. Uno de los centros de referencia constituye el Centro de Especialidades Dermatológicas ubicado en el Hospital General de San Lorenzo donde acuden pacientes de varias regiones sanitarias. Otros centros de referencia para el diagnóstico y seguimiento de la enfermedad son el Hospital Menonita de la Ciudad de Itacurubí de la Cordillera, el Hospital de Clínicas y el Hospital Nacional de Itauguá. Las Unidades de Salud de la Familia llevan adelante el Programa de Lepra en sus comunidades; no obstante, muchos pacientes acuden a los centros de referencia para no ser reconocidos en las localidades donde viven como pacientes de lepra según datos de este estudio.

Los pacientes fueron inicialmente identificados mediante la revisión de fichas clínicas en el Centro de Especialidades Dermatológicas. De esta forma se identificaron 65 personas que habían culminado el tratamiento farmacológico en el período establecido. Se trató de contactar con ellos telefónicamente, pero 17 no respondieron o los números fueron cambiados. Todo el proceso se realizó con el acompañamiento de personal del Programa Nacional de Control de Lepra. 
Finalmente, fueron convocados 48 pacientes para la presentación del estudio, la firma del documento de conformidad y la descripción de las actividades a realizar. De estos 48 pacientes, 44 aceptaron participar del estudio en la entrevista realizada en los consultorios del Centro de Especialidades Dermatológicas. Los cuatro pacientes que se negaron a participar lo hicieron por sentirse invadidos en su privacidad o por temor a que los familiares y vecinos conocieran su problema de salud. De los 44 , fueron nuevamente excluidos 10 pacientes, debido a que las viviendas estaban ubicadas en localidades con difícil acceso, causado por caminos de tierra deteriorados por lluvias constantes, barrios o asentamientos muy inseguros con presencia de jóvenes adictos a las drogas, por traslado de domicilio, motivos personales, incomodidad ante la presencia del personal sanitario, horarios laborales muy largos, poco tiempo de permanencia en el domicilio o cuestionarios muy extensos. La muestra final estuvo integrada por 34 pacientes: 9 casos y 25 controles.

\begin{tabular}{|c|c|c|c|}
\hline Características & $\begin{array}{c}\text { Casos } \\
n=9(\%)\end{array}$ & $\begin{array}{c}\text { Control } \\
n=25(\%)\end{array}$ & $\begin{array}{c}\text { Total } \\
\mathrm{n}=34(\%)\end{array}$ \\
\hline $\begin{array}{l}\text { Edad - años (media } \pm D E) \\
\quad<20 \\
20-39 \\
40-59 \\
\geq 60\end{array}$ & $\begin{array}{c}- \\
2(22,2) \\
3(33,3) \\
4(44,4)\end{array}$ & $\begin{array}{r}1(4) \\
4(16) \\
11(44) \\
9(36)\end{array}$ & $\begin{array}{r}53 \pm 15,2 \\
1(2,9) \\
6(17,6) \\
14(41,2) \\
13(38,2)\end{array}$ \\
\hline $\begin{array}{l}\text { Sexo } \\
\text { Masculino } \\
\text { Femenino }\end{array}$ & $\begin{array}{l}7(77,7) \\
2(22,2)\end{array}$ & $\begin{array}{l}12(48) \\
13(52)\end{array}$ & $\begin{array}{l}19(55,9) \\
15(44,1)\end{array}$ \\
\hline $\begin{array}{l}\text { Estado civil } \\
\text { Casado/unión libre } \\
\text { Soltero } \\
\text { Separado/divorciado/viudo }\end{array}$ & $\begin{array}{l}5(55,5) \\
1(11,1) \\
3(33,3)\end{array}$ & $\begin{array}{r}17(68) \\
6(24) \\
2(8)\end{array}$ & $\begin{array}{r}22(64,8) \\
7(20,6) \\
5(14,8)\end{array}$ \\
\hline $\begin{array}{l}\text { Nivel educativo } \\
\text { Ninguno } \\
\text { Primario } \\
\text { Secundario } \\
\text { Universitario }\end{array}$ & $\begin{array}{l}1(11,1) \\
5(55,5) \\
3(33,3) \\
-\end{array}$ & $\begin{array}{r}1(4) \\
13(52) \\
8(32) \\
3(12)\end{array}$ & $\begin{array}{c}2(5,9) \\
18(53) \\
11(32,3) \\
3(8,8)\end{array}$ \\
\hline $\begin{array}{l}\text { Situación laboral } \\
\text { Empleado con salario fijo* } \\
\text { Trabajador independiente** } \\
\text { Ama de casa } \\
\text { No trabaja } \\
\text { Trabajo ocasional***}\end{array}$ & $\begin{array}{l}2(22,2) \\
3(33,3) \\
2(22,2) \\
1(11,1) \\
1(11,1)\end{array}$ & $\begin{array}{l}6(24) \\
4(16) \\
7(28) \\
4(16) \\
4(16)\end{array}$ & $\begin{array}{l}8(23,5) \\
7(20,6) \\
9(26,4) \\
5(14,7) \\
5(14,7)\end{array}$ \\
\hline
\end{tabular}

*Funcionario público, docente, cajera, empleado de empresa constructora, guardia de seguridad. **Trabajo doméstico, herrería, carpintero, gomería, técnico en informática, agricultor. ${ }^{* * *}$ Conductor de carrito, flete.
Se realizaron entrevistas domiciliarias con cada uno de los pacientes utilizando cuestionarios semiestructurados, que incluyeron características sociodemográficas como la edad, sexo, estado civil, nivel educativo y situación laboral. Otras variables estudiadas fueron características clínicas como el tipo de discapacidad, clasificación de la enfermedad (clasificación operativa según la OMS y la clasificación de Madrid), número de profesionales consultados para el diagnóstico de lepra y retraso en el diagnóstico.

Se definió como retraso en el diagnóstico, si el paciente tardaba más de doce meses entre la aparición de los primeros síntomas y el diagnóstico de lepra por parte del médico que prescribió el tratamiento farmacológico. La clasificación operativa de la enfermedad (OMS) establece dos categorías, la multibacilar, paciente con más de cinco manchas y dos o más nervios afectados y la paucibacilar, paciente con menos de cinco manchas y un solo nervio afectado $^{1}$. La clasificación de Madrid emplea parámetros clínicos, inmunológicos, baciloscópicos, histopatológicos, que incluyen dos tipos polares de mayor a menor respuesta inmune: el lepromatoso y tuberculoide, así como otros dos tipos, uno de inicio de la lepra, indeterminado y bordeline, casos de inmunología dudosa o difusa ${ }^{13}$. Las formas clínicas bordeline y lepromatosa suelen presentar las llamadas reacciones leprosas que se pueden producir antes del tratamiento farmacológico, downgranding, durante e incluso después de finalizar el tratamiento upgranding. Estas reacciones deben ser controladas por personal médico a fin de evitar el deterioro de la función nerviosa $a^{1,10,14}$.

\section{Resultados}

Se incluyeron en el estudio 34 pacientes, provenientes de cinco distritos de Paraguay, nueve pacientes conformaron los casos, de los cuales seis presentaron discapacidad G1 al diagnóstico, de estos, tres tenían falta de sensibilidad en manos y antebrazos y, tres de ellos presentaban ulceraciones de evolución crónica, en talones y dorso de los pies al momento de las visitas domiciliarias. Dichas lesiones aún no habían sido evaluadas por personal médico.

De los tres pacientes con discapacidad G2 al diagnóstico, dos tenían deformidad en una de las manos, un paciente presentaba ulceración en la planta del pie y deformación en ambas manos.

La edad media de los pacientes fue $53 \pm 15,2$ años, el $55,9 \%$ fue de sexo masculino, el $64,8 \%$ estaba casado o vivía en unión libre con su pareja y 5,9\% no accedió a la educación formal. En cuanto a la ocupación, 23,5\% de los pacientes percibía un salario fijo, el 26,4 \% correspondía a amas de casa y $20,6 \%$ era trabajador independiente; $14,7 \%$ de los pacientes estaba desempleado al momento del estudio y un porcentaje igual realizaba trabajos ocasionales (Tabla 1). 


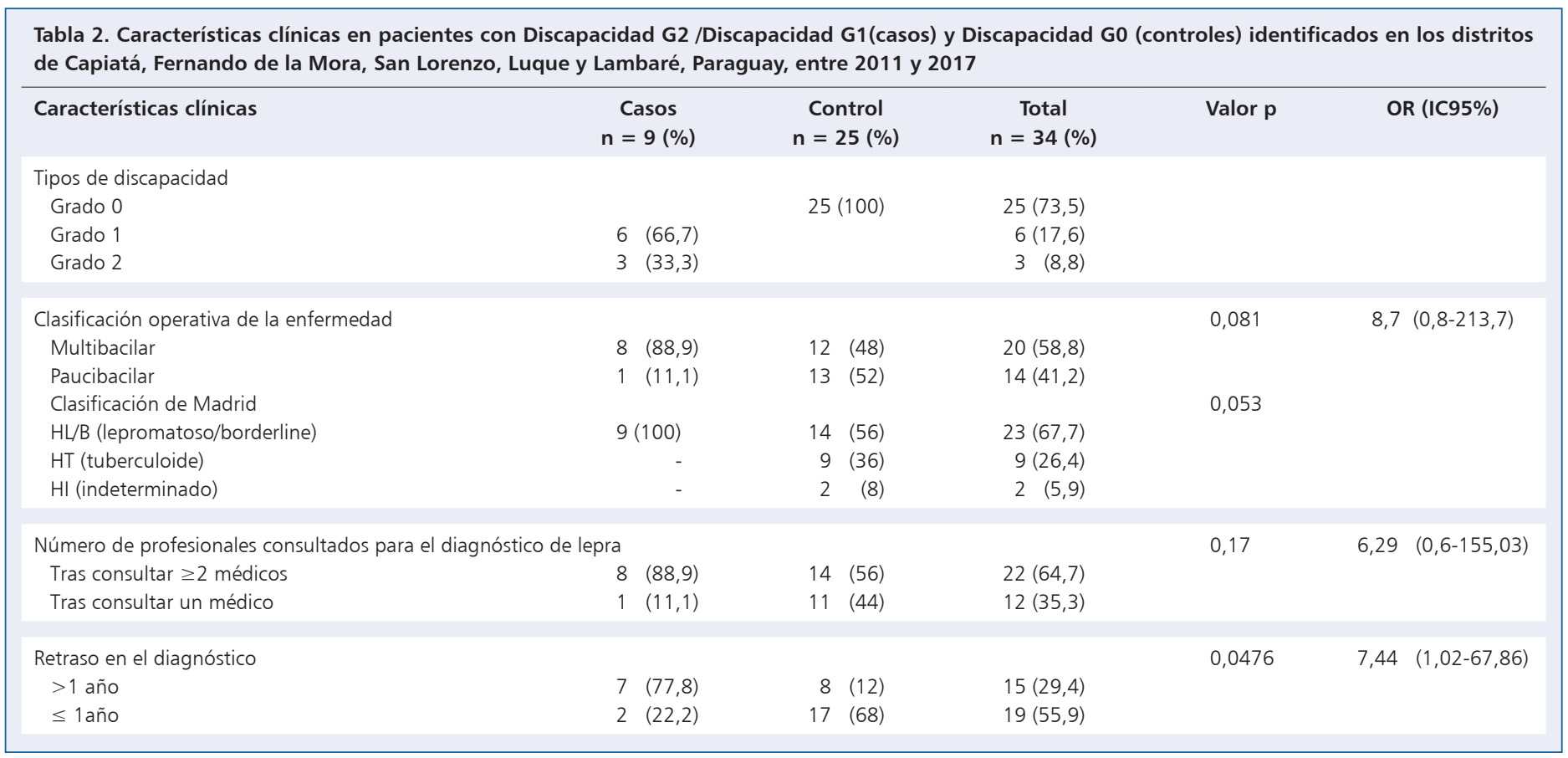

En relación a las características clínicas, el 58,8\% de los pacientes presentó lepra multibacilar, 88,9\% de los casos en comparación al $48 \%$ de los controles $(\mathrm{p}=0,081)$. El polo lepromatoso estuvo presente en $67,7 \%$ de los pacientes, $100 \%$ de los casos versus $56 \%$ de los controles $(\mathrm{p}=0,053)$. En relación al número de profesionales consultados para el diagnóstico de lepra como indicador de la dificultad del diagnóstico de la enfermedad, el 64,7\% de los pacientes fue diagnosticado luego de consultar con dos o más médicos, $88,9 \%$ entre los casos comparado al $56 \%$ entre los controles $(\mathrm{p}=0,17)$. El retraso en el diagnóstico, como factor pronóstico de discapacidad mostró diferencia significativa entre los dos grupos, así se observó que el tiempo hasta el diagnóstico fue mayor a un año en $77,8 \%$ de los casos comparado al $12 \%$ de los controles ( $p=0,047$; OR: 7,44, IC95\%: 1,02-67,86) (Tabla 2).

\section{Discusión}

Se identificó como factor pronóstico de discapacidad G1 y G2, el retraso en el diagnóstico. El 77,8\% de los casos había sido diagnosticado en un tiempo mayor a un año tras la aparición de los síntomas comparado al 12\% del grupo control. Estos resultados evidencian lo mencionado por varios autores, siendo considerado el retraso en el diagnóstico de la lepra como factor pronóstico de discapacidad $^{7}$. El tiempo de retraso como factor pronóstico de discapacidad presenta diferentes definiciones según los autores. Así, Para Srinivas y cols., un retraso mayor a cuatro meses ya se relaciona con discapacidad en pacientes de cinco estados de India ${ }^{16}$. Chu y cols., en China, informaron que 48,9\% de los casos con discapacidad G2 tuvo un retraso mayor a 12 meses, en comparación a 28,5 en los pacientes sin discapacidad G2 y Kumar y cols., en India, mencionaron que un retraso por encima de 36 meses se relacionó con el desarrollo de discapacidad. Van Veen y cols., recomiendan limitar el inicio del tratamiento dentro de los seis meses del inicio de los síntomas para reducir la probabilidad de desarrollo de la discapacidad. La proporción de pacientes diagnosticados después de este plazo se podría utilizar, según algunos estudios, como un indicador operativo de calidad deficiente de los servicios del programa de control de lepra de un país ${ }^{5,6,15}$. La detección temprana de los casos es conocida por ser el factor más importante en la prevención de discapacidad por lepra ${ }^{5}$.

El retraso en el diagnóstico resulta de una compleja interacción entre factores físicos, sociales, económicos, y psicológicos ${ }^{16}$. El retraso no tiene la misma influencia en la evolución de la enfermedad en todos los pacientes, puesto que intervienen varios factores como el tipo de lepra, la inmunidad del paciente, el inicio del tratamiento, los cuidados durante el tratamiento, las reacciones leprosas que pudiera sufrir el paciente y el número de nervios afectados al momento del diagnóstico. Cada paciente debe ser abordado en forma individual y objetiva ${ }^{1,17}$.

Esta tardanza en el diagnóstico ha sido investigada 
considerando dos factores, el primero debido al paciente y el segundo debido al sistema sanitario. Así, Mutuvel y cols., en India, determinaron mediante un estudio de casos y controles, que en los casos, en promedio se tuvo un retraso debido al paciente de 14,8 $\pm 19,5$ meses y debido al sistema de salud de 8,4 \pm 9,6 meses y, en los controles 7,2 $\pm 16,7$ y $3,9 \pm 5,8$ meses, respectivamente ${ }^{18}$. Sumados los dos retrasos, la media \pm SD en los casos fue $23,2 \pm 21,9$ meses, que resultó significativamente mayor $(\mathrm{p}<0,01)$ que los controles $(11,1 \pm 18,9 \text { meses })^{18}$.

En el presente estudio, no se consideró por separado, el retraso debido al paciente o debido sistema sanitario; sí fue considerada la dificultad para el diagnóstico en términos del número necesario de profesionales para el diagnóstico de la enfermedad e inicio del tratamiento. El 64,7\% de los pacientes fue diagnosticado luego de consultar con dos o más médicos, que si bien fue mayor entre los casos que en los controles ( 88,9 vs $56 \%$ ), no alcanzó a una diferencia significativa.

Los pacientes recurren a médicos especialistas luego de haber consultado con varios profesionales, probablemente por desconocimiento sobre la enfermedad o por el estigma y temor que provoca la lepra. Si bien no se han realizado estudios sobre el estigma que provoca la lepra en Paraguay, existen referencias de trabajos en otros países. Mutuvel y cols., describieron que los pacientes no acuden a los servicios de salud por suponer que los síntomas no son graves, o por miedo al estigma y la discriminación relacionados a la lepra ${ }^{18}$. En este trabajo no se presentan los resultados sobre el conocimiento o estigma relacionado a la enfermedad de los pacientes, aspectos que serán presentados en otro artículo.

En el Paraguay, los centros del servicio público de salud que tienen personal capacitado y con amplia experiencia en el diagnóstico de la lepra son: el Hospital Menonita, Hospital de Clínicas que es un hospital-escuela de la Universidad Nacional de Asunción, el Centro de Especialidades Dermatológicas y el Hospital Nacional de Itauguá. Los profesionales médicos de los citados centros y de las Unidades de Salud de la Familia de los distritos donde se realizó el estudio manifestaron que podría existir poca experiencia en el diagnóstico de la lepra en los servicios, ya que se la considera una enfermedad eliminada.

La reducción de la carga de la enfermedad sigue siendo una meta en los países con alta carga de lepra ${ }^{10,11}$. La OMS describió los servicios de calidad en la lepra, citando los aspectos del abordaje del tratamiento, donde se incluyen la prevención de discapacidades, la rehabilitación, el apoyo psicológico y socioeconómico de los pacientes y sus familias ${ }^{19}$.

Aunque en este estudio no se analizó la posible diferencia en el retraso en el diagnóstico entre hombres y mujeres, sí informado por otros estudios ${ }^{5}$, es destacable mencionar la mejor actitud de las mujeres con respecto a la consulta médica, que también se pone de manifiesto en la población en el Paraguay; las mujeres tienden a acudir más frecuentemente al médico que los hombres. Sin embargo, en este estudio hubo predominio de sexo masculino entre los casos, lo cual coincide con los estudios realizados en Brasil e India ${ }^{5,16}$. Esto podría explicarse porque los hombres están más expuestos, por realizar el trabajo fuera de la casa o podría también explicarse por un factor genético de protección en el sexo femenino, aspecto estudiado por Alvarenga y cols. No obstante, cuestiones relacionadas a los mecanismos y rutas que guían esos efectos, así como el rol de las hormonas sexuales no son claros aún ${ }^{20}$.

La forma multibacilar fue predominante como lo manifestaron otros estudios ${ }^{10}$, lo cual indica indirectamente el retraso en el diagnóstico ${ }^{5}$. Los pacientes afectados por esta forma constituyen el reservorio de la enfermedad, que implica la continua posibilidad de contagio a los contactos intradomiciliarios y a otros contactos cercanos, ya sea en el trabajo o el entorno del vecindario. Este indicador permite identificar focos activos de transmisión de lepra, que deberían ser priorizados para las acciones de vigilancia y control. Además, permite caracterizar el comportamiento clínico de la lepra en las diferentes entidades territoriales y la oportunidad del diagnóstico ${ }^{19}$. Hay creciente evidencia de posible transmisión subclínica de la enfermedad en países donde la lepra es endémica; muchos pacientes no reconocen un contacto cercano con algún caso conocido de lepra ${ }^{21}$.

Según protocolos aplicados en Paraguay, los pacientes paucibacilares deberían acudir a control médico una vez al año, por dos años, luego de la terapia farmacológica, y los multibacilares durante los cinco años posteriores para realizar evaluaciones sobre el daño neural y controlar las reacciones leprosas ${ }^{22}$. Los pacientes acudían para el seguimiento a los centros de referencia citados anteriormente y manifestaron temor para recurrir al servicio de su distrito para evitar que la información llegara a oídos de sus vecinos o parientes; muchos enfermos ocultaban su patología a sus parientes cercanos, incluso con quienes convivían a causa del temor a ser discriminados, según manifestaron. El Programa Nacional de Control de la Lepra ha descentralizado las actividades de diagnóstico y entrega de medicamentos a las Regiones Sanitarias, lo cual posibilita que los pacientes recurran a los profesionales en su departamento y puedan acceder al tratamiento farmacológico gratuito; por otra parte, el temor a los posibles gastos en movilidad o alimentación hace que los pacientes, según sus propias manifestaciones, dilaten la consulta. Esto podría retrasar su diagnóstico y tratamiento precoz.

Existen aún muchas interrogantes con relación al mecanismo de transmisión. No todos los contactos de multibacilares desarrollan lepra, esto podría deberse a otros factores como aspectos nutricionales mencionados por Oktaria y cols. ${ }^{23}$. No siempre es conocida la fuente de 
puede desarrollar esta dolencia, aunque no haya sido notificado oficialmente.

Los pacientes en este estudio se ocupaban en general de actividades laborales sin ingresos fijos o actividad de ama de casa, y tenían bajo nivel educativo. Concordamos con estos autores que los bajos ingresos económicos pueden dificultar el acceso a la salud en los pacientes en esos países, como estaría ocurriendo en el Paraguay ${ }^{5,12,16}$.

Silva y cols., han estudiado factores socioeconómicos asociados con un incremento de riesgo de contraer lepra, como la dificultad de acceso a condiciones de vivienda, agua y salud que contribuirían al desarrollo de la enfermedad ${ }^{24}$. Estos antecedentes pueden conducir a la manifestación de las consecuencias de la enfermedad en pacientes en situación de pobreza, sin acceso a servicios básicos y servicios de salud ${ }^{12}$. En Paraguay, ante similares hallazgos expuestos, se puede suponer que los mismos factores socioeconómicos influyen en el desarrollo de la lepra y podrían conducir al empeoramiento del estado de salud de los pacientes ${ }^{25}$

El bajo nivel educativo de los pacientes diagnosticados con lepra ${ }^{10}$, significaría un mayor riesgo de contraer la enfermedad ${ }^{24}$. En el presente estudio, los pacientes con discapacidad, en su mayoría alcanzaron sólo nivel educativo primario, lo que se relaciona con el déficit de acceso a la educación verificado en pacientes con lepra en otros estudios. Chu y cols., verificaron analfabetismo en pacientes con Discapacidad ${ }^{25}$. En Brasil, un estudio mostró que niveles bajos de instrucción se correspondían con dos veces mayor riesgo de desarrollar lepra ${ }^{24}$.

La mayoría de los pacientes en los dos grupos tenía más de 40 años, hallazgo similar a lo reportado por estudios, lo cual pone de manifiesto el largo período de incubación de la enfermedad, que puede llegar a los 20 años $1,7,10,20$

La necesidad de la prevención de discapacidades descrita por la OMS, se puso de manifiesto en los pacientes del grupo casos que experimentaron limitación de su actividad laboral, debieron realizar cambios para evitar riesgos por su discapacidad o incluso, llegaron al desempleo. Sufrieron limitación social por causa de deformidad en las manos o lesiones crónicas en los pies, temor al rechazo por sus amistades u otras personas. Las actividades domésticas como cocinar, utilizar artefactos eléctricos, o trabajar en el jardín, también fueron limitadas ya que podrían ser causa de lesiones por falta de sensibilidad en manos o antebrazos originando quemaduras o cortes. Las lesiones en los pies se empeoraban si no eran adecuadamente desinfectadas, y protegidas todos los días. La falta de descanso ocasionaba hinchazón y dolores molestos en los pacientes. A pesar de los problemas persistentes muchos pacientes no acudían a los controles médicos, ya fuese por motivos económicos o cuestiones laborales. Esto implica la necesidad de un enfoque 
multidisciplinario por parte del sistema de sanitario a fin de posibilitar el empoderamiento de los pacientes de su propia salud.

Los pacientes que constituyeron el grupo control no presentaban discapacidad al momento del estudio, pero es sabido que luego del tratamiento, los pacientes $\mathrm{PB}$ o MB podrían presentar daño neural, y por ende, discapacidad. En el desarrollo de la discapacidad intervienen factores como retraso en el diagnóstico, inicio de tratamiento y compromiso neural, si lo hubiere al diagnóstico ${ }^{8}$. Todo lo expuesto contribuye a que la prevalencia de discapacidad sea muy variable entre los diferentes estudios ${ }^{7}$.

La discapacidad producida por la lepra puede afectar la calidad de vida del paciente, inducir temor, estigma, discriminación y limitación de su actividad social; de ahí la importancia de evitar esta consecuencia o tratarla adecuadamente si estuviera presente. Los servicios de salud deberían proporcionar al paciente la posibilidad de recuperación y reinserción social ${ }^{5,10}$.

Una limitación fundamental de este estudio fue el tamaño de muestra, debido a que no fue posible acceder a todos los pacientes diagnosticados en el período de estudio. Muchos pacientes no pudieron ser contactados por cambios de número telefónico o domicilio, lo cual imposibilitó las entrevistas. Otros pacientes se negaron a participar del estudio porque mantenían en secreto su enfermedad, no todos sus familiares sabían que ellos habían recibido tratamiento contra la lepra.

A pesar de las limitaciones, en este estudio se puso de manifiesto que el retraso en el diagnóstico podría ser un factor pronóstico de discapacidad. Los factores socioeconómicos que están relacionados con la lepra deben ser estudiados en el país a fin de brindar parámetros para la utilización de políticas públicas encaminadas a reducir la carga de lepra. ES preciso investigar las variables relacionadas a la transmisión que han permitido el mantenimiento de la incidencia de la lepra en el Paraguay, a pesar de que los esfuerzos han conducido en algunas regiones sanitarias a alcanzar la meta de prevalencia propuesta por la OMS.

Agradecimientos: A los pacientes por colaborar en el trabajo. A Investigación para el Desarrollo por el apoyo administrativo.

\section{Referencias bibliográficas}

1.- White C, Franco-Paredes C. Leprosy in the $21^{\text {st }}$ century. Clin Microbiol Rev. 2015; 28(1): 80-94 doi: 10.1128 / CMR.00079-13.

2.- Saliba C, Ísper A, Oliveira M, Saliba T, Jefferson R. El estigma y el prejuicio de la lepra: influencia en la condición humana. Rev Soc Bras Med Trop. 2015; 48(2): 194-201. doi: 10.1590/0037-8682-0004-2015.

3.- OMS/OPS. Plan de acción para acelerar el logro de la eliminación de la lepra en América Latina y el Caribe. 2011 64p. [cited 2019 December 21] Disponible en: https://www. paho.org/hq/dmdocuments/2014/2014-chaplan-accion-eliminacion-lepra-lac.pdf.

4.- OMS. Weekly Epidemiological Record, 30 August 2019, vol. 94, 35/36. pp. 389-412. Disponible en: https:/www.who.int/wer/2019/ wer9435_36/en/

5.- Chu T, Liu D, Huai P, Chen X, Han S, Chen $\mathrm{S}$, et al. Comprehensive measures succeeded in improving early detection of leprosy cases in post-elimination era: Experience from Shandong province China. PLoS Negl Trop Dis. 2020; 14(2): e0007891 https://doi. org/10.1371/journal.pntd.0007891.

6.- Freitas L, Duarte E, García L. Tendencias de los principales indicadores de lepra en municipios brasileños con alto riesgo de transmisión de la lepra 2001-2012. BMC Infect Dis. 2016; 16(1): 472. https://doi.org/10.1186/s12879-016-17982.

7.- Kumar A, Girdhar A, Girdhar B. Riesgo de desarrollar discapacidad en el tratamiento previo y posterior a la terapia con múltiples fármacos entre la lepra multibacilar: estudio de cohorte. Br Med J Open 2012; 2:e000361. doi: 10.1136 / bmjopen-2011-000361.

8.- Van Brakel W. Neuropatía periférica en la lepra y sus consecuencias. Lepr Rev. 2000; 71: 14653. doi: 10.5935/0305-7518.20000086.

9.- Fava V, Manry J, Cobat A, Orlova M, Van T, Moraes M, et al. A genome wide association study identifies a lncRna as risk factor for pathological inflammatory responses in leprosy. PLoSGenet. 2017; 13(2):e1006637. https://doi. org/10.1371/journal.pgen.1006637.

10.- Manzan B, Castro S, Rodrigues L. Limitación de actividad y restricción de participación social en relación a rango de edad, sexo y educación en personas con lepra. An Bras Dermatol. 2017; 92(3): 335-9. doi: 10.1590/ abd1806-4841.20175216.

11.- Moreira J, Strina A, Silva J, Menezes L, Freitas D, Penna M, et al. Socioeconomic risk markers of leprosy in high-burden countries: A systematic review and meta-analysis. PLoS Negl Trop Dis. 2018; 12(7). e0006622. doi: 10.1371/journal.pntd.0006622.

12.- Proenda A B, Lyon S, Grossi M, Da Costa M. Factores de riesgo de discapacidad física al salir de la terapia con múltiples fármacos en nuevos casos de lepra en un centro de referencia en Brasil. Rev. Inst Med Trop S. Paulo. 2019; 61: e13:1-9. doi:https://www. scielo.br/pdf/rimtsp/v61/1678-9946-rimtsp61-S1678-9946201961013.pdf.
13.- Guerrero E, Martínez F, Atoche C, Arrazola J, Guzmán R. Lepra. Clasificación y cuadro clínico Dermatol Rev Mex 2012; 56(1): 47-54 Disponible en: https://www.medigraphic.com/ pdfs/derrevmex/rmd-2012/rmd121g.pdf.

14.- Serrano H, Cardona N. Neuropatía leprótica: una mirada integral de la afección periférica causada por Mycobacterium leprae. Salud Uninorte 2017; 33 (3): 451-63. doi: http:// dx.doi.org/10.14482/sun.33.3.10926.

15.- Nicholls P, Croft R, Richardus J, Withington $\mathrm{S}$, Smith W. Delay in presentation, an indicator for nerve function status at registration and for treatment outcome: the experience of the Bangladesh Acute Nerve Damage Study cohort. Lepr Rev. 2003; 74: 349-56. doi:10.47276/ 1r.74.4.349.

16.- Srinivas G, Muthuvel T, Lal V, Vaikundanathan K, Schwienhorst-Stich E, Kasang C. Risk of disability among adult leprosy cases and determinants of delay in diagnosis in five states of India: A case-control study. Plos Negl Tropl Dis. 2019; 13(6): e0007495. doi: https://doi. org/10.1371/journal.pntd.0007495.g003.

17.- Van Veen N, Meima A, Richardus J. The relationship between detection delay and impairment in leprosy control: A comparison of patient cohorts from Bangladesh and Ethiopia. Lepr Rev 2006; 77(4): 356-65. https://pubmed. ncbi.nlm.nih.gov/17343222/\#article-details.

18.- Mutuvel T, Srinivas G, Petros I, Hemant D, Vasudek R, Rajbir S, Sanjeev K. "Perdí 3 años pensando que no era un problema": Retrasos del paciente y del sistema de salud en el 
diagnóstico de lepra en India: un estudio de métodos mixtos. Plos Negl Tropl Dis 2017; 11(1): e0005192. https://doi.org/10.1371/ journal.pntd.0005192.

19.- OMS/OPS. Estrategia mundial para la lepra 2016 2020. 2017 p 82 [citado el 21 de diciembre de 2019]. Disponible en :https://apps.who.int/iris/bitstream/hand le/10665/250119/9789290225591-Spa. pdf? sequence $=10$.

20.- Alvarenga N, Rocha G, Lana F. The TLR1 gene is associated with higher protection from leprosy in women. PloSOne 2018; 13(10): e0205234.https://doi.org/10.1371/ journal.pone. 0205234 .
21.- Goulart I, Bernardes D, Marques C, Pimenta V, Goncalves M, Goulart L. Risk and protective factors for leprosy development determined by epidemiological surveillance of household contacts. Clin Vaccine Immunol. 2008; 15(1): 101-5. doi: 10.1128/CVI.00372-07.

22.- OPS. Ministerio de Salud de Costa Rica. Normas para la Atención Integral y Control de la Enfermedad de Hansen en Costa Rica. 2012 p 100 [Consultado el 21 de julio de 2020] Disponible en: https://www.binasss.sa.cr/ protocolos/hansen.pdf.

23.- Oktaria S, Huriff N, Naim W, Bing H, Nijsten R, Richardus J. Dietary diversity and poverty as risk factors for leprosy in Indonesia: A case-control study. PloS Negl Trop Dis. 2018; 13: 12(3): e0006317. doi: 10.1371/journal. pntd.0006317.

24.- Silva J, Tamond A, Moreira J, Alves A, Strina A, Ichihara M, et al. Determinantes socieconómicos de la detección de nuevos casos de lepra en la cohorte de 100 millones de brasileños: un estudio de vinculación poblacional. 2019; 7: e1226-e1236. https://doi. org/10.1016/S2214-109X(19)30260-8.

25.- Wagenaar I, van Muiden L, Alam K, Bowers R, Hossain MA, Kispotta K., et al. Diet-related risk factors for leprosy: a case-control study. PLoS Negl Trop Dis. 2015; 9(5): e0003766. doi: 10.1371/ journal.pntd.0003766.

\section{ALIENTO DE VIDA*}

Dieron recepción al paciente en la emergencia, el cuadro clínico era claro COVID-19, un paciente de dos años de edad daba sus últimos respiros. Un corte eléctrico agotó la esperanza de que sobreviviera el pequeño, pero mi querido colega era de esos que nunca nos rendimos. Cuando el niño dejó de respirar... Plutarco se olvidó de las precauciones y le comenzó a dar respiración boca a boca, Juancito como por milagro comenzó a respirar nuevamente, llegó la electricidad, se le colocó respirador artificial y sobrevivió. Su aliento revivió al niño, pero Plutarco tres semanas después no sobrevivió.

RUFINITO

*Primer Premio en el Concurso de Cuentos: "Yo, el COVID y la Seguridad del Paciente en 100 palabras". Fundación para la Seguridad del Paciente, Chillán, Ñuble, Chile. 2021. Reproducido con autorización de los Editores 